\title{
Myxoid liposarcoma in a 91-year-old patient
}

\author{
Brandon S Sheffield ${ }^{*}$ and Torsten O Nielsen
}

\begin{abstract}
Background: Myxoid liposarcoma is a mesenchymal malignancy most commonly presenting in young adults. This tumor is known for its characteristic chromosomal rearrangement at the DDIT3 locus.

Results: We report a case of myxoid liposarcoma in a 91-year-old, the oldest known patient with this disease-entity. FISH analysis of the DDIT3 and FUS loci demonstrate the pathognomonic chromosomal alteration in the setting of predominantly round cell histology on biopsy, confirmed by RT-PCR.

Conclusion: Myxoid liposarcoma affects mostly young adults but can be seen in the elderly population. Molecular and cytogenetic assays are helpful auxiliaries to histology in the setting of unusual histology and clinical presentation.
\end{abstract}

Keywords: Liposarcoma, Myxoid liposarcoma, Round cell liposarcoma, Advanced age, DDIT3, FUS, FISH

\section{Background}

Myxoid liposarcoma, the second-most common subtype of liposarcoma, accounts for $10 \%$ of soft tissue sarcomas [1]. Myxoid liposarcoma tends to occur on extremities, with two thirds of cases originating in the thigh [2]. This disease affects a younger demographic than most cancers with peak incidence between ages 30-50 [1], and is the most common subtype of liposarcoma in the pediatric age group [3].

Typical myxoid liposarcoma histology shows nonpleomorphic ovoid mesenchymal cells and rare lipoblasts amidst a prominent myxoid stroma, with an intricately plexiform vasculature. Round-cell liposarcoma, a highgrade form of myxoid liposarcoma, lacks myxoid matrix leaving closely packed, round mesenchymal cells. Regions of myxoid and round-cell liposarcoma morphologies often coexist (MLS/RCLS).

Both morphologies share identical cytogenetic aberrations and are thus considered as a single pathologic entity [1]. A reciprocal translocation $t(12 ; 16)$ can be identified in the majority of cases [4] creating an in-frame fusion of FUS and DDIT3 that is specific for MLS/RCLS [5]. A small proportion of cases harbor an alternate $t(12 ; 22) E W S-D D I T 3$ rearrangement [6]. Demonstration of disruption at the DDIT3 locus is an effective diagnostic tool for MLS/RCLS [7], with break-apart fluorescent in situ hybridization (FISH) probes commercially available.

The most powerful prognostic marker in MLS/RCLS is the presence of a round cell component comprising greater

* Correspondence: Brandon.s.Sheffield@gmail.com

Department of pathology and laboratory medicine, University of British

Columbia, 899 West 12th Avenue, Vancouver, British Columbia V5Z 1M9, Canada than $5 \%$ of the tumor. These high grade round cell liposarcaromas have been shown to behave more aggressively with decreased overall survival and metastasis free survival [8].

Compared with other soft tissue sarcomas, MLS/RCLS is particularly sensitive to radiation therapy [9]. Accordingly, neoadjuvant radiation has been recommended in the treatment of myxoid liposarcoma [10]. This finding places increased significance on accurate diagnosis prior to surgical resection.

The following report depicts a case of MLS/RCLS in a rare elderly age demographic while highlighting the salient diagnostic features of this disease, and the value of molecular testing in the context of unusual clinical features and histology.

\section{Case presentation}

A 91-year-old male presented to a local emergency department complaining of a large mass in the medial thigh that had grown in size over the preceding several weeks. The mass was otherwise asymptomatic.

The patient's past medical history included atrial fibrillation and hypertension, prostate cancer treated by resection with local recurrence treated by radiotherapy 20 years previously, laryngeal cancer treated by radiotherapy 14 years previously, and finally, separate histories of both colon and anal cancer treated with an abdominal-perineal resection 6 years previously. The patient reported a family history of ovarian cancer and multiple prostate cancers.

CT scan of the pelvis demonstrated a $19 \mathrm{~cm}$ lobulated mass with complex septations and central necrosis in the adductor compartment of the left thigh (Figure 1). 


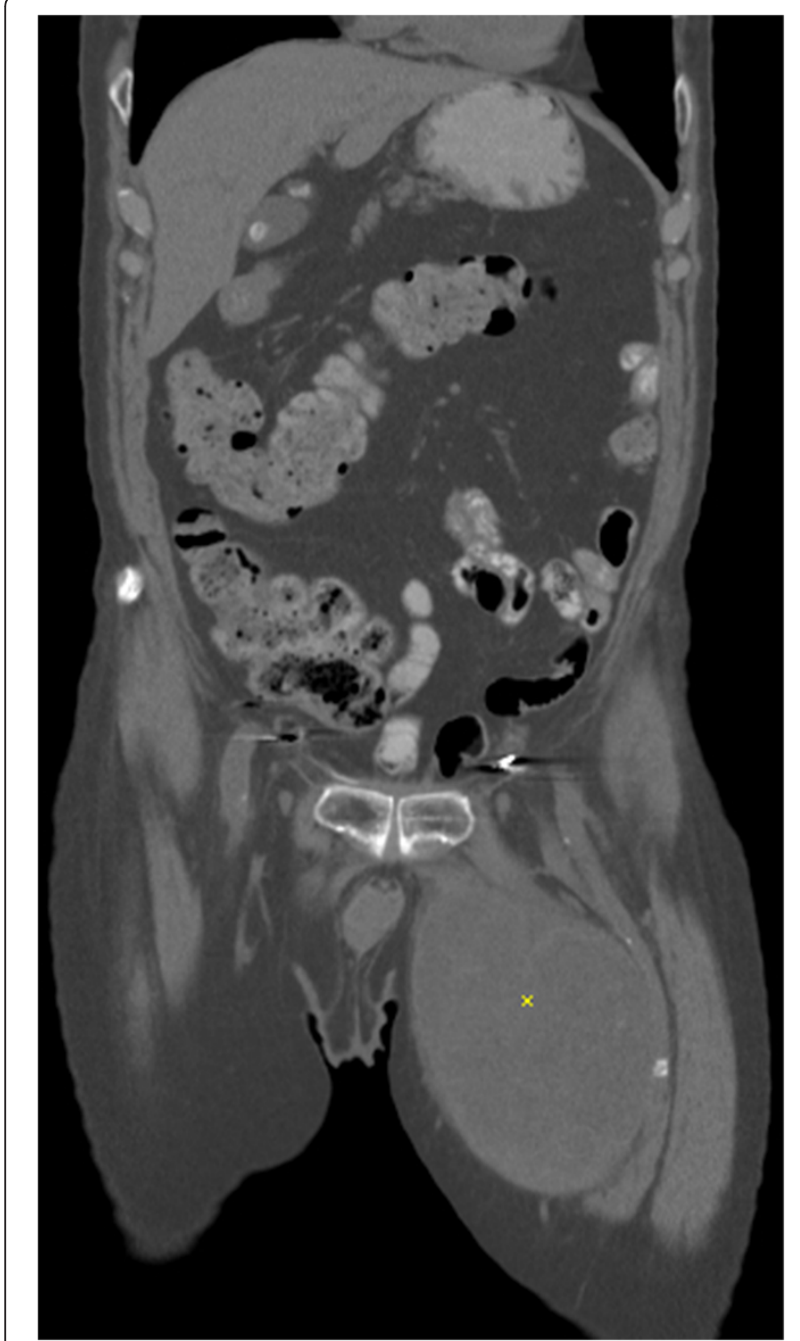

Figure 1 Abdomino-pelvic CT scan showing tumor in the left medial thigh $(*)$.
Core needle biopsy of the mass under ultrasound guidance showed high-grade spindled, ovoid and round cell morphology. Although a small amount of myxoid matrix and complex vasculature were noted, no unequivocal lipoblasts were evident and the tumor was considered to lack differentiation towards any specific lineage (Figure 2a). Immunohistochemical workup was nonspecific, with the tumor showing positive staining for S-100 and weak staining for CD34 and BCL-2. No immunoreactivity was present for melan-A, pankeratin, desmin, smooth-muscle actin, TLE, CD20, CD43, or CD99. Mitotic figures were numerous and the Ki67 index was high. With no specific diagnosis evident after this workup, in the context of blue cell histology for a large soft tissue mass, FISH analysis was performed for DDIT3, FUS, and EWSR1. Break-apart probes for DDIT3 and FUS demonstrated chromosomal rearrangements at both of these loci (Figures 2b, c), while EWSR1 was intact. RT-PCR confirmed expression of a type 1 FUSexon7-DDIT3exon2 fusion transcript. The RT-PCR was performed according to previously established protocols [11], using oligonucleotide primers to exon 6 of FUS ( $5^{\prime}$-gaacccagaggtcgtggag-3') and exon 2 of DDIT3 (5'-tgctttcaggtgtggtgatg-3').

Based on the pathognomonic molecular findings, a diagnosis of MLS/RCLS was made. The patient was discussed at multidisciplinary tumor board, and offered neoadjuvant radiation treatment followed by surgical excision. Both radiation and surgery were welltolerated. Final pathology showed a $19 \mathrm{~cm}$ lobulated heterogenous yellow and hemorrhagic mass (Figure 3a). Microscopic sections showed a mixture of high-grade round cell areas similar to that seen at biopsy, as well as regions showing a more typical myxoid liposarcoma histology that had not been evident on the core biopsy (Figures 3b, c), providing morphological confirmation of the diagnosis.

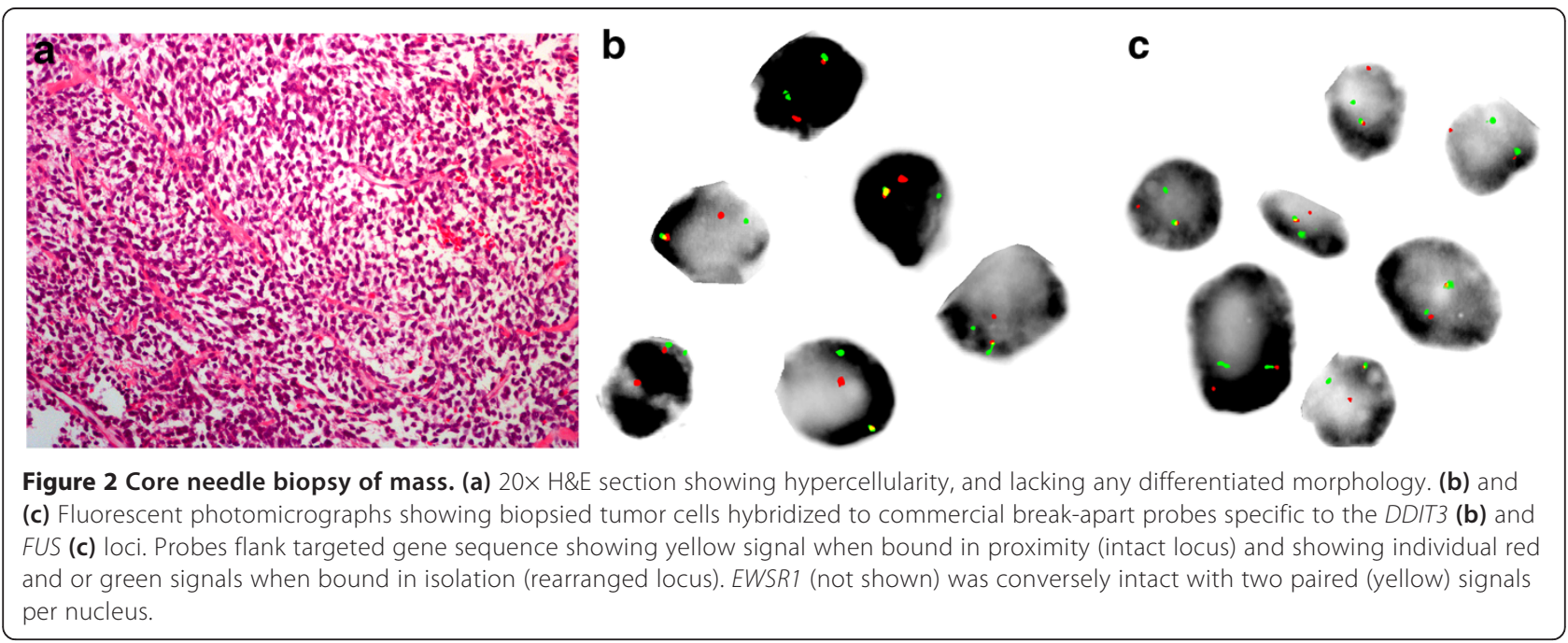



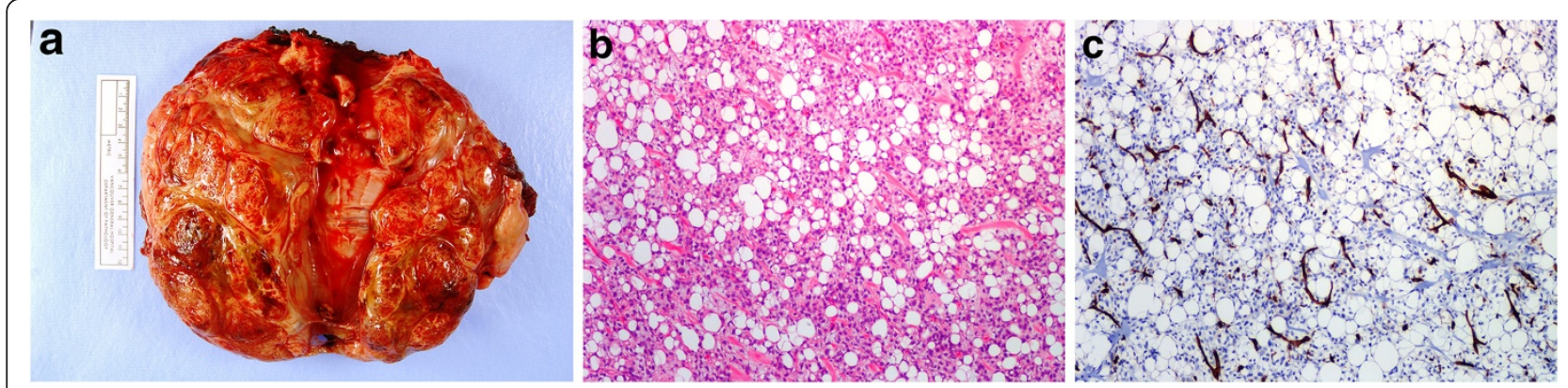

Figure 3 Excision of mass. Gross photo showing $19 \mathrm{~cm}$ yellow, hemorrhagic mass (a), featuring regions with typical myxoid liposarcoma, 10x H\&E (b), and typical chicken-wire vasculature highlighted by CD31 immunohistochemical stain (c).

A search of the medical literature was performed for reports of myxoid and/or round cell liposarcoma in the elderly. Moreau et al. [10] recently published a series of $418 \mathrm{MLS} /$ RCLS patients, the oldest being 85 years old at presentation. To our knowledge, the current case represents the oldest reported age for a patient presenting with primary myxoid or round cell liposarcoma.

\section{Discussion}

Several facets of myxoid liposarcoma diagnosis and treatment are highlighted in this case. The diagnosis of myxoid liposarcoma in an elderly patient is uncommon, but does occur. Thus an advanced age should not exclude myxoid liposarcoma when other clinical, radiologic, histologic, or molecular findings support this diagnosis.

Myxoid liposarcoma can show a wide variation of histologic patterns, at times within the same tumor [12]. This can lead to difficult diagnoses, especially on small biopsy specimens. This case highlights the value of molecular diagnosis as an adjunct to histology. FISH is available in specialty centers, and is available to most surgical pathology labs as it can be performed on standard formalin-fixed, paraffin-embedded tissue sections (shipped to central labs if needed). In the current era of diagnostics, molecular confirmation of sarcomas is quickly becoming a standard of care for several types of sarcomas bearing pathognomonic genetic events. The availability of molecular diagnostic tools may, in part, contribute to the overall improved outcomes observed in sarcoma patients treated at higher volume institutions [13].

The accurate preoperative diagnosis of myxoid liposarcoma prioritized neoadjuvant radiotherapy as the next step in management. This treatment modality has particular benefit in myxoid liposarcoma [9]. Accurate diagnosis on biopsy in elderly patients may have additional value if poor baseline functioning precludes the patient as a surgical candidate, as palliative radiation may be particularly effective after a diagnosis of myxoid liposarcoma.

\section{Conclusion}

Myxoid/round cell liposarcoma is a relatively common sarcoma among younger adults presenting with a large, deep-seated malignant primary soft tissue mass. However, this tumor can affect older individuals. Molecular cytogenetic studies can greatly assist diagnosis in cases with unusual clinical and histologic features.

\section{Consent}

Written informed consent was obtained from the patient for publication of this case report and any accompanying images. A copy of the written consent is available for review by the Editor-in-Chief of this journal.

\section{Competing interests}

The authors declare that they have no competing interest.

\section{Authors' contributions}

BSS Assisted in interpreting the diagnostic biopsy, compiled the case report, and wrote the manuscript. TON Issued pathological diagnosis of the biopsy and resection specimens, performed cytogenetic and molecular workup, edited the manuscript, and supervised the project. Both authors read and approved the final manuscript.

\section{Acknowledgements}

We would like to acknowledge Marina Pacheco and Angela Goytain for assistance with the gross photography and molecular testing. Molecular studies were supported by funding from the Liddy Shriver Sarcoma Initiative. We would like to thank the UBC Anatomical Pathology residency program for their continued support.

Received: 5 September 2013 Accepted: 23 October 2013 Published: 19 November 2013 
1. Antonescu C, Ladanyi M: Myxoid liposarcoma. In Pathology and genetics of tumours of soft tissue and bone. Edited by Fletcher CDM, Unni KK, Mertens F. Lyon: IARC Press; 2002:40-43 [Kleihues P and Sobin LH (Series Editors): World Health Organization classification of tumors].

2. Moore Dalal K, Kattan MW, Antonescu CR, Brennan MF, Singer S: Subtype specific prognostic nomogram for patients with primary liposarcoma of the retroperitoneum, extremity, or trunk. Ann Surg 2006, 44(3):381-391.

3. Alaggio R, Coffin CM, Weiss SW, Bridge JA, Issakov J, Oliveira AM, Folpe AL: Liposarcomas in young patients: a study of 82 cases occurring in patients younger than 22 years of age. Am J Surg Pathol 2009, 33(5):645-658.

4. Sreelantaiah C, Karakousis CP, Leong SPL, Sandberg AA: Cytogenetic findings in liposarcoma correlate with histopathologic subtypes. Cancer 1991, 69:2484-2495.

5. Antonescu CR, Elahi A, Humphrey M, Lui MY, Healey JH, Brennan MF, Woodruff JM, Jhanwar SC, Ladanyi M: Specificity of TLS-CHOP rearrangement for classic myxoid/round cell liposarcoma. J Mol Diagn 2000, 2:132-138.

6. Aman P, Panagopoulos I, Lassen C, Fioretos T, Mencinger M, Toresson H, Hoglund M, Forster A, Rabbits TH, Ron D, Mandahl N, Mitelman F: Expression patterns of the human sarcoma-associated fenes FUS and EWS and the genomic structure of FUS. Genomics 1996, 37:1-8.

7. Narendra S, Valente A, Tull J, Zhang S: DDIT3 Gene break-apart as a molecular marker for diagnosis of myxoid liposarcoma- assay validation and clinical experience. Diagn Mol Pathol 2011, 20(4):218-224.

8. Antonescu CR, Tschernyavsky SJ, Decuseara R, Leung DH, Woodruff JM, Brennan MF, Bridge JA, Neff JR, Goldblum JR, Ladanyi M: Prognostic impact of p53 status, TLS-CHOP fusion transcript structure, and Histologic grade in myxoid liposarcoma: a molecular and clinicopathologic study of 82 cases. Clin Cancer Res 2001, 7:3977-3987.

9. Chung PWM, Deheshi BM, Ferguson PC, Wunder JS, Griffin AM, Catton CN, Bell RS, White LM, Kandel Ram O, Sullivan B: Radiositivity translates into excellent local control in extremity myxoid liposarcoma. Cancer 2009, 115:3254-3261.

10. Moreau LC, Turcotte R, Ferguson P, Wunder J, Clarkson P, Masri B, Isler M, Dion N, Werier J, Ghert M, Deheshi B: Myxoid/round cell liposarcoma (MRCLS) revisited: an analysis of 418 primarily managed cases. Ann Surg Oncol 2012, 19:1081-1088.

11. Powers MP, Wang WL, Hernandez VS, Patel KS, Lev DC, Lazar AJ, Lopez-Terrada DH: Detection of myxoid liposarcoma-associated FUS-DDIT3 rearrangement variants including a newly identified breakpoint using an optimized RT-PCR assay. Mod Pathol 2010, 23(10):1307-1315.

12. Fritchie KJ, Goldblum JR, Tubbs RR, Sun Y, Carver P, Billings SD, Rubin BP The expanded histologic spectrum of myxoid liposarcoma with an emphasis on newly described patterns. Am J Clin Pathol 2012, 137:229-239.

13. Gutierrez JC, Perez EA, Livingstone AS, Franceschi D, Koniaris LG: Should soft tissue sarcomas be treated at high-volume centers? An analysis of 4205 patients. Ann Surg 2007, 245(6):952-958.

\section{Submit your next manuscript to BioMed Central and take full advantage of:}

- Convenient online submission

- Thorough peer review

- No space constraints or color figure charges

- Immediate publication on acceptance

- Inclusion in PubMed, CAS, Scopus and Google Scholar

- Research which is freely available for redistribution

Submit your manuscript at www.biomedcentral.com/submit
Biomed Central 